\title{
Improving nurse documentation and record keeping in stoma care
}

\author{
Lesley Law, Karen Akroyd, Linda Burke
}

\begin{abstract}
Evidence suggests that nurse documentation is often inconsistent and lacks a coherent and standardized approach. This article reports on research into the use of nurse documentation on a stoma care ward in a large London hospital, and explores the factors that may affect the process of record keeping by nursing staff. This study uses stoma care as a case study to explore the role of documentation on the ward, focusing on how this can be improved. It is based on quantitative and qualitative methods. The medical notes of 56 patients were analysed and in addition, focus groups with a number of nurses were undertaken. Quantitative findings indicate that although $80 \%$ of patients had a chart filed in their medical notes, only a small portion of the form was completed by nursing staff. Focus group findings indicate that this is because forms lacked standardization and because the language used was often ambiguous. Staff also felt that such documentation was not viewed by other nurses and so, was not effective in improving patient care. As a result of this study, significant improvements have been made to documentation used on the stoma care ward. This is an important exploration of record keeping within nursing in the context of the Nursing and Midwifery Council's emphasis on the importance of documentation in achieving effective patient outcomes.

Key Words: Nursing - Documentation - Record keeping - Stoma care
\end{abstract}

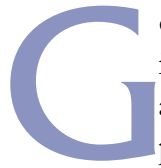

ood record keeping is an important aspect of a nurse's role and an essential element in good patient care. If documentation is not completed satisfactorily, communication between groups of staff can be adversely affected and, if patient records are not read, care can be compromised (Health Service Ombudsman, 2000). There is also an important legal dimension as in law it is deemed that care was not undertaken if no records exist (Nursing and Midwifery Council (NMC) 2009). Current evidence suggests that much nurse documentation lacks structure and allows important information to be lost

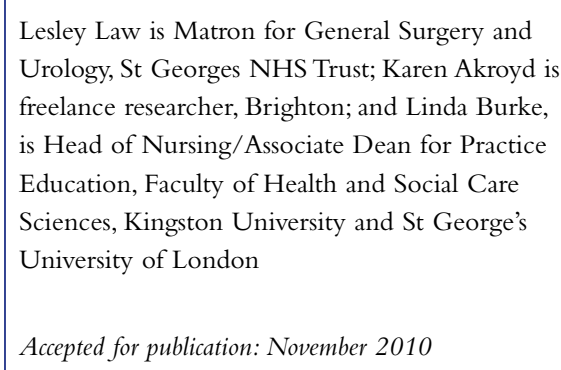

to the reader (Irving et al, 2006). Written information is often not read by members of the multidisciplinary team, and loses its significance in the planning of future care. The NMC recently introduced new guidelines in which it emphasizes the need for good documentation (NMC, 2009):

'Good record keeping is an integral part of nursing and midwifery practice, and is essential to the provision of safe and effective care. It is not an optional extra to be fitted in if circumstances allow.'

This article reports on research into the use of nurse documentation on a stoma care ward in a large London hospital, and explores the factors that may affect the process of record keeping by nursing staff. This study is important because improved record keeping by nurses will ultimately lead to an improvement in patient care. Stoma care is used as a case study example, although findings are relevant to nurse documentation in general. As a result of this study, significant improvements have been made to the documentation used on the stoma care ward. This is an important study in the context of the NMC's emphasis on the importance of documentation in achieving effective patient outcomes.

\section{Background}

The NMC (2009) emphasizes the need for good nurse documentation and have established guidelines to help promote good practice. Records should enable care and progress to be communicated to the wider multi-professional team, and have the ability to record any problems and/or changes in the patient's condition (NMC, 2009). Ideally, nurse documentation should replicate the format adopted by each individual trust. At present, there is no single model or template for patient records; however, the NHS is aiming to standardize and improve communication by implementing a single, unified and agreed format (NMC, 2006).

Currently the responsibility lies with individual trusts to agree a standard format for documentation, and this can lead to across each trust (McGeehan, 2007). Effective nurse documentation should include the date and time of observations and interactions, and need to be written as soon after the event as possible (Royal College of Nursing (RCN), 2003). Documentation may be used to defend a nurse's actions in a court of law and considerable reliance is placed on records in such a hearing (RCN, 2003). Poor record-keeping by nurses is a feature of many of the complaints to the Health Service Ombudsman (2000) and may result in compromised patient care owing to poor communication. The RCN (2003) has published guidelines specifically for colorectal and stoma care nursing. The aim of the study was to establish whether stoma care documentation needed to be changed to help improve patient information.

The project on which this article is based was undertaken within the stoma care department and two acute surgical wards of a large London teaching hospital [AQ1: dates of project?]. This articles reports on a study exploring the role of documentation 


\section{STOMA CARE}

on the stoma care ward, focusing on how this can be improved. Findings have resulted in improvements being made to documentation used on the ward.

\section{Literature review}

Complaints to the NHSfrequently and persistently focus on issues of poor communication, deficiencies in nursing care documentation and poor record-keeping. (Tingle, 2002; Abraham, 2003). A review of the literature suggests that the documenting of nursing care is a complex and often repetitive process. Mann and Williams (2003) argue that the standardization of nursing documentation can lead to a direct benefit in patient care. O'Conner et al (2007) have supported this argument in a recent initiative to improve nurse documentation in a trust where at least four different documentation formats were in use. They found that the introduction of a standardized format to all nursing documentation had a positive effect on its completion, and consequently improved both communication and patient care.

A number of other studies point to such a lack of structure within documentation (Friberg et al, 2005; Hyde et al, 2005; Irving et al, 2006). Allen (1998) suggests that the nursing record has become little more than an elaborate accounting mechanism in which nurses list interventions to serve only as documented evidence for a court of law if needed. Other studies point to the extent of repetition in nursing documentation. Both Cheevakasemsook et al (2006) and Taylor (2003) describe how data, including information on vital signs and progress reports, were recorded on different forms and were often incomplete. Martin et al (1999) and Payne et al (2000) point to the need for a more concise, non-duplicating system of documentation. Payne et al (2000) found that much nursing documentation hides the work carried out by nurses. Similarly, studies by Hyde et al (2005) and Friberg et al (2005) suggest that the emotional labour involved in nursing, such as listening, teaching and advocating on the patient's behalf tend not to be documented. It is argued that because nurses find it difficult to articulate a caring experience into words, they tend to record the tasks undertaken, thereby undervaluing the work they actually do.

A number of other studies point to the retrospective nature of nurse documentation. Hardy et al (2000) argue that most forms of nursing documentation, including care plans and progress reports, were not used to inform nursing care. Rather than refer to documentation, nurses preferred to communicate verbally, using their own 'scraps' of paper which held important information that would not be open to scrutiny by others. This approach did not arise from a desire for more paperwork, but from the perceived inadequacies of ward documentation. Clemow (2006) found that patient documentation was rarely referenced during 'handover', despite significant efforts to complete it on time at the end of each nursing shift. Sexton et al (2004) found that $84.6 \%$ of information conveyed by nurses during handover could have been recorded in writing. However, Payne et al (2000) argue that nursing handovers form an important part of nurse's socialization, as well as the need to communicate information.

Clemow (2006) and Hardy et al (2000) suggest that, unlike other health professionals, who record their observations in the knowledge that their assessments will be read and acknowledged by others, nurses do not. O'Connell et al (2000) found that care plans and documentation not specific to individual specialties or patients are usually out of date and therefore of little value. Many studies indicate that, despite the problems discussed, nurses viewed it as an important aspect of their work (Allen, 1998; Martin et al, 1999; Hardy et al, 2000; Friberg et al, 2005; Cheevakasemsook et al, 2006; Irving et al, 2006). Three studies cite lack of time as a factor affecting the documentation process (McKenna et al, 2000; Cheevakasemsook et al, 2006; Kalisch, 2006). NMC Guidelines for Record and Record Keeping (2009) indicate that nursing records should be consistent, clearly written and accurate. They also support the principle of a shared patient record in which individuals make their own entries to communicate patient progress to the wider team. A review of the literature therefore suggests that nurse documentation often fails to meet these guidelines.

\section{Methodology}

The research aims were to explore how documentation was completed by ward and stoma care staff and to establish the factors that may affect the completion of the stoma care forms. It was hoped that qualitative findings would lead to an insight into what the users of the form considered both important and unimportant aspects of the present stoma care documentation, and whether they considered it helpful in their practice. It was also expected that findings would be transferable to other clinical areas in terms of the maintenance of good documentation and how this can be improved.

The study population was chosen from two surgical wards within an acute trust, both of which are regular users of the present stoma care chart. In addition, the staff from the Stoma Care Department were also involved in the study. The sampling method used was a non-probability purposive sample (Morrell and Harvey, 1999). Both wards were 26-bed surgical units, which at any one time may have five or more patients with stomas. The patient sample consisted of 56 patients who had stoma surgery within the last year and were inpatients. Both elective and emergency cases were included to give an overall representative picture. Names of appropriate patients were accessed via the stoma care database. Case notes were obtained from the main hospital records department.

Ethical issues were discussed with the Clinical Audit Office of the host trust and it was decided that in line with Research Governance Framework (Department of Health (DH), 2001), the dignity, rights and wellbeing of participants must be of primary consideration. Permission, approval and consent for the project was obtained from ward managers as the research had the potential to cause distress to staff, who may feel their practice is being scrutinized and possibly criticized. It was important to reassure nursing staff that the aim of this project was to evaluate the stoma care chart to improve the quality of care through more suitable documentation and not a study to describe the quality of patient care. Confidentiality and anonymity was assured, and all data collected was kept securely. [AQ2: how was this ensured?] All participants were assured that all discussions and transcripts would remain anonymous.

\section{Data collection}

The research design included both quantitative and qualitative methods. The medical notes of 56 patients who had had stoma surgery within the last year were analysed to determine how patient information was being documented. Ward-based documentation is kept at the patient's bedside and is completed by both the ward nurses and the clinical nurse specialist in stoma care. Electronic patient records are also held in the stoma care department, which include a patient progress report. Nurses were invited to document their observations of the stoma and to complete a 'check list' to be used in conjunction with a nursing care plan to provide a coordinated approach to progressive teaching. The check list comprised a set of 
criteria of what constitutes an effective chart based on expert opinion from the literature and ward-based opinion.

In addition, focus groups were undertaken with a total of 14 nurses to explore the issues surrounding stoma care documentation and record-keeping. The idea behind these questions was to assist in establishing factors that may affect the completion of the form and gain insight into what the users of the form considered both important and unimportant aspects of the present stoma care documentation, and whether or not they considered it helpful in their practice. Five interviews were planned and each group lasted approximately 20 minutes, conducted by the author. [AQ3: any particular author? or all three?] The maximum number of participants present at each interview was three. The same questions were asked of both the ward-based and stoma care nurses in order to obtain an overall picture of the use of the documentation. Open-ended questions were used to allow participants an opportunity to express their views in their own words. Focus groups were considered the most effective way of gathering information on the use and effectiveness of the chart, as they may help to clarify, explore or confirm ideas and thoughts from the actual users of the form (Goodman and Evans, 2006).

Focus group findings were analysed using thematic analysis in which key issues were identified and then validated against subsequent comments, and checked against the literature. Documentary analysis was based on the following criteria from the literature (Owen, 2005; O’Conner et al, 2007):

- Accessibility

- Enables progress and the recording of changes detected and/or problems to be communicated to the multi-professional team

- Clear without the use of jargon or abbreviations

- Dated and timed

- Structured and standardized to an agreed recognizable format

- Does not duplicate information documented on other charts.

\section{Findings}

The results reveal that although $80 \%$ of patients had a chart filed in their medical notes, only a small portion of the form was completed by nursing staff. Slightly more than half $(53 \%)$ of the patient sample has no documented information relating to the type of operation they had undergone and consequently, no information on type of stoma. This information is important in terms of stoma care because nursing staff need this to determine the usual appearance of the stoma and the type of faecal output expected.

The first section of the chart, which invites both ward and stoma nurses to comment on the appearance and output of the stoma for the first 7 postoperative days, show no entries by ward nurses on $35 \%$ of the charts and no entries by stoma nurses on 30\% of the charts. Similar results emerge from the 'appliance change' section of the form: $45 \%$ of charts have no entries in this section by ward nurses, and $60 \%$ have no entries by stoma care nurses. Similar results are found in the 'steps to discharge' section: 55\% of forms have no entries in this section by ward nurses and 25\% have no entries by stoma care nurses. Fourty-five percent of charts have between 1 and 3 days completed by the ward nurses and $70 \%$ of charts have between one and three days completed by the stoma care nurses.

With regard to focus group findings, most respondents commented on the importance of nurse documentation in general, and are keen to ensure they are able to complete this. Most respondents place a high value on the importance of documenting when the appliance was last changed and whether or not the stoma was active. One nurse makes the following comment, which is representive of the views of many others:

'The form is useful for the staff to ascertain when the bag was last changed. We can then document whether their bowel are active. I would like a section for us to complete when we change the bag.'

A number of respondents find much of the language used in nurse documentation ambiguous. A number of respondents suggest that this ambiguity made them cautious about documenting their findings because they are worried about using incorrect terminology. One respondent suggested that 'you should have a description or picture of what it should look like rather than using these words'.

As well as finding the language ambiguous, some participants also indicated that they find the process of documentation a little daunting. As one nurse explains:

'I feel our knowledge is being tested and am afraid $I$ will get the answers wrong, so I tend to either not fill out that part or copy what was written above.'
Most respondents express concern about the large amount of information requested and say that they find this confusing. Many therefore suggest a reduction in the number of questions for each section of the form to avoid duplication. Many respondents feel that if the format of the stoma care documentation was similar in format to other nurse documentation, it is more likely to be completed.

Many participants are unsure whose role it is to complete the stoma care form and others are unclear about who the information on the chart is for. One respondent suggests that the form should be used by the stoma nurses to provide the ward nurses with instructions on what to do next with the patient. Most respondents feel that the chart is not generally read by health professionals, implying that this was a reason why it is often not completed. The following comment is indicative of the view of many respondents:

'If I thought anybody was going to use or read the chart I would be

more inclined to write on it.'

Interestingly, although focus group respondents in the stoma care team give similar answers to the other group, they thought that the form was largely not used by anyone other than the immediate stoma care team.

\section{Discussion}

Findings from this study indicate that stoma care forms were not being fully completed by nursing staff because a lack of standardization was causing confusion, and form completion was time-consuming. This issue was raised by Mann and Williams (2003) who found that a lack of standardization acted as a significant barrier to effective documentation and record-keeping within nursing. Findings from the focus groups suggest that if documentation was simplified to follow a standard format, nurses would be more likely to complete it. O'Conner et al (2007) also found this to be the case.

Participants in this study also felt that duplication of information was a particular problem, leading to reluctance to complete the forms thoroughly. These findings are reflected by Cheevakasemsook et al (2006) and Owen (2005), who argue that nurse documentation and record-keeping is often improved by a reduction in duplication.

Results also suggest that ward nurses had a problem with the ambiguous language used on documentation and that this prevented them from formally recording their findings. 
As the chart was often not completed, it became redundant to nursing practice, which created an additional barrier to its use. Many respondents believed that the documentation they completed would not be read by other health professionals. This is synonymous with evidence from Hardy et al (2000), who found that nurses lacked the confidence to document their findings formally, and felt that documentation would not be referred to by colleagues on the ward.

One of the aims of this research was to use the findings to implement a change in practice and changes to stoma care documentation have therefore been implemented on the ward. Specifically, nurse documentation has been rewritten and standardized to comply with the other documentation used within the trust. Comments from focus group participants were taken into account in order to improve the format and design of stoma care forms, and to generate a more effective use of the form. It was considered appropriate to reduce the form in size, and to request information less frequently. As ward staff indicated that they needed clarification concerning the usual appearance of a stoma and information about some common complications, a 'flow chart' was added to the documentation to help staff understand what was required. To help avoid ambiguous language, the form was adapted to include an explanation of the terminology used in stoma care. This is also important in the context of NMC guidelines (2009), which advise against using jargon or ambiguous language in patient records.

It is acknowledged that change can cause anxiety to nursing staff (Swage, 2000), and support and guidance was provided throughout the change to achieve smooth implementation. This included an educational programme for staff and an information pack for future use. The literature points to the need for a standardized, jargon-free method of documentation which avoids duplication and is easy to complete. The changes implemented on the stoma care ward as a result of this study's findings will have a direct impact on the effective completion of documentation and will improve patient care as a result.

\section{Limitations}

One of the limitations of the study was that although the data obtained from the audit of stoma care forms were valid, as this was simply a collection of data it would have been more reliable if the quality of the written data on each form was analysed and commented on in more detail. A comparison was not made with other nursing documents, which may have established whether they were completed. Another limitation was that, as this is a case study, findings cannot be generalized. However, readers may be able to identify useful information from the findings in this study that could apply to their own areas of practice.

\section{Conclusions}

The results of this study reflect much of the evidence suggesting that nurse documentation, although viewed by nurses as important, is inadequately completed and often not used to inform practice. The new documentation is now being used on the ward as a result of this study and has been well received by nursing staff. This research will form part of a continuous cycle of evaluation and development for nurses caring for patients with a stoma.

The stoma care department has now started an educational programme with some positive feedback from many nurses who have attended. Important changes have been made to nurse documentation, leading to an improvement in patient care. These improvements to practice reflect the findings of the literature review, which indicated that poor documentation procedures mean that nursing staff often fail to complete forms, and that patient care can be adversely affected.

The changes implemented reflect NMC (2009) guidelines, which aim to promote good practice and will help staff communicate issues of patient care to the wider multi-professional team. It is acknowledged that for the transference of knowledge and skills to be effective, educational training must be constantly readdressed and re-evaluated. (Griscti and Jacono, 2006). Therefore, teaching days are held for staff every 3 months in which all nurses have an introduction to stoma

[AQ5; please add 3-5 key points, in full sentences, summarizing the main learning points of the article]

care and related terminology. To date, these sessions have been well evaluated by attendees. It is important that nurse documentation continues to be reviewed and improved on in order to ensure high quality patient care.

\section{Conflict of interest: [AQ4: please state]}

Abraham A (2003) Inadequate nursing care and the failure to keep adequate records. Prof Nurse 18(6): 347-9

Allen D (1998) Record keeping and routine practice: the view from the wards. J Adv Nurs 27(6): 1223-36

Cheevakasemook A, ChapmanY, Francis K, Davies C (2006) The study of nursing documentation complexities. Int J Nurs Pract 12(6): 366-74

Clemow R (2006) Care plans as the main focus of nursing handover: information exchange model. $J A d v$ Nurs 15(11): 1463-5

Doherty L (2009) Using 'coded expressions of sarcasm' or 'humorous abbreviations' to describe patients has been ruled out in Nursing and Midwifery Council (NMC) guidance on record-keeping. Nursing Standard [AQ6: not cited in main body - please add or remove from this list. If added, please provide issue number and page numbers]

Frank-Stromborg M, Christensen A, Elmhurst D (2001) Nurse documentation: not done or worse done the wrong way-part 1. Oncol Nurs Forum 28(4): 697-802 [AQ7: not cited in main body - please add or remove from this list

Friberg F, Bergh A, Lepp M (0000) In search of details of patient teaching in nursing documentation-an analysis of patient records in a medical ward in Sweden. J Clin Nurs 15(12): 1550-8

Goodman C, Evans C (2006) The research in nursing practice. In: Gerrish K,d Lacey A eds. Blackwell Publishing [AQ8: please provide main book title],

Hardy M, Payne S, Coleman P (2000) 'Scraps': hidden nursing information and it's influence on the delivery of care. JAdv Nurs 32(1): 346-50

Health Service Ombudsman (2000) Fifth report for session 1999-2000 Selected cases and summaries of completed investigations October 1999-March 2000. The Stationary Office, London

Hyde A, Treacy M, Scott A et al (2005) Modes of rationality in nursing documentation: biology, biography and the 'voice of nursing'. Nurs Inq 12(2): 66-8

Irving K, Treacy M, Scott A, Hyde A, Butler M, MacNeela $\mathrm{P}$ (2006) Discursive practices in the documentation of patient assessments. J Adv Nurs 53(2): 151-9

Kalisch BJ (2006) Missed nursing care: a qualitative study. $J$ Nurs Care Qual 21(4): 306-13

Mann R, Williams J (2003) Standards in medical record keeping. Clin Med 3(4): 329-21

Martin A, Hinds C, Felix M (1999) Documentation practices of nurses in long term care. J Clin Nurs 8(4): $345-52$

McGeehan R (2007) Best practice in record-keeping. Nurs Stand 21(17): 51-5

Morrell C, Harvey G (1999) The Clinical Audit Handbook: Improving the quality of health care. Bailliere Tindall, China

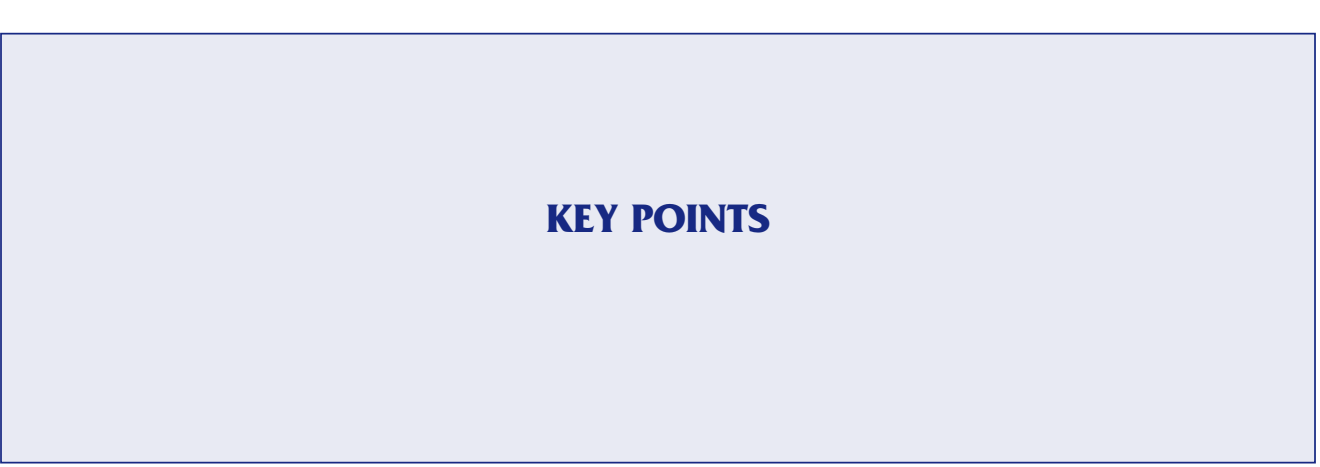


Nursing and Midwifery Council (2009) Record keeping:: Guidance for nurses and midwives. http://tinyurl. com/3xxs7p9 (accessed 8 November 2010)

Nursing and Midwifery Council (2005) Guidelines for Records and Record Keeping. http://tinyurl. com/3xydjuj (accessed 8 November 2010)

O'Connell B, Myers H, Twigg D, Entriken F (2000). Documenting and communicating patient care: Are nursing care plans redundant? Int J Nurs Pract 6: 276-80

O'Conner K, Earl T, Hancock P (2007) Introducing improved nursing documentation across a trust. Nurs Times 103(6): 134-7

Owen K (2005) Documentation in nursing practice. Nurs Stand 19(32): 48-9

Payne S, Hardey M, Coleman P (2000) Interactions between nurses during handover in elderly care. J $A d v$ Nurs $\mathbf{3 2}(2)$ : 277-85

Price B (2006) Teaching good record keeping. Nurs Stand 20(22): 34-6

Royal College of Nursing (2003) Documentation in Colorectal and Stoma Care Nursing: RCN guidance for nursing staff. http://tinyurl.com/3ydm5av (accessed 8 November 2010)

Sexton A, Chan C, Elliott M, Stuart J, Jayasuriya R, Crookes P (2004) Nursing handovers: do we really need them? J Nurs Manag 12(1): 37-42

Taylor H (2003) An exploration of the factors that affect nurses record keeping. Br J Nurs 12(12): 751-8

Tingle J (2002) Health professionals keep making the same mistakes. Br J Nurs 11(7): 414

Tucker A (2009) Improved Record Keeping with Reading handovers. Nursing Manag 16(8): 30-4 [AQ9: not referenced in main body - please add or remove from this list]

[AQ10: references missing for:

McKenna et al 2000

DH 2001

Swage 2000

Grisch and Jacono 2006]

[AQ11: might it be relevant to include a section discussing the impact of better record-keeping on patients and overall patient care?] 